\title{
Low-temperature dynamics of magnetic colloids studied by time-resolved small-angle neutron scattering
}

\author{
A. Wiedenmann, ${ }^{2, *}$ U. Keiderling, ${ }^{1}$ M. Meissner, ${ }^{1}$ D. Wallacher, ${ }^{1}$ R. Gähler, ${ }^{2}$ R. P. May, ${ }^{2}$ S. Prévost, ${ }^{3}$ M. Klokkenburg, ${ }^{4}$ \\ B. H. Erné, ${ }^{4}$ and J. Kohlbrecher ${ }^{5}$ \\ ${ }^{1}$ Hahn-Meitner-Institut, Structure Research, Glienicker Straße 100, D-14109 Berlin, Germany \\ ${ }^{2}$ Institut Laue-Langevin, Boîte Postale 156, F-38042 Grenoble, Cedex 9, France \\ ${ }^{3}$ Stranski-Laboratorium für Physikalische und Theoretische Chemie, Technical University Berlin, Straße des 17 Juni 124, \\ Sekretariat TC 7, D-10623 Berlin, Germany \\ ${ }^{4}$ Van't Hoff Laboratory for Physical and Colloid Chemistry, Utrecht University, Padualaan 8, NL-3584 CH Utrecht, The Netherlands \\ ${ }^{5}$ Laboratory for Neutron Scattering, ETH Zurich \& Paul Scherrer Institut, CH-5232 Villigen PSI, Switzerland
}

(Received 28 January 2008; revised manuscript received 18 March 2008; published 15 May 2008)

\begin{abstract}
The dynamics of ordering and relaxation processes in magnetic colloids has been studied by means of stroboscopic small angle neutron scattering techniques in an oscillating magnetic field. Surfactant stabilized ferrofluids (FFs) of $\mathrm{Fe}_{3} \mathrm{O}_{4}$ and $\mathrm{Co}$ nanoparticles have been investigated as a function of temperature and frequency and compared to a solid $\mathrm{Cu}$ alloy with 0.8 vol \% Co precipitates. This technique allowed elucidating the dynamical nature of the locally ordered domains in both ferrofluids as "living objects" becoming arrested below the freezing temperature of the solvent. The time-dependent intensities have been analyzed in terms of Langevin statistics including dynamical interparticle structure factors, which scale with the square of the Langevin function. The local ordering is mainly determined by the effective dipole-dipole interaction, which is enhanced by the partial alignment of the particle moments in an external magnetic field. Starting from the frozen state, the amount of freely rotating particle moments increases continuously with increasing temperature. The dynamical structure factors describing the hexagonal $\left(\mathrm{Fe}_{3} \mathrm{O}_{4}\right.$ - $\left.\mathrm{FF}\right)$ or chainlike $(\mathrm{Co}-\mathrm{FF})$ ordering reach a maximum around the melting temperature. The alignment of particle moments along the applied field is governed by the fast Brownian rotation of individual nanoparticles and small aggregates, while the magnetic relaxation of longer dipolar chains and local hexagonal domains is much slower. In contrast, no field-induced interparticle correlations occur in the diluted solid $\mathrm{CuCo}$ alloy where the moment relaxation is purely of fast Néel type, which—due to a low anisotropy constant—follows the oscillating field at all temperatures.
\end{abstract}

DOI: 10.1103/PhysRevB.77.184417

PACS number(s): 75.10.-b, 61.05.F-, 47.65.Cb

\section{INTRODUCTION}

Magnetic single-domain nanoparticles in colloidal dispersions spontaneously form dipolar chains in head-to-tail conformation, as predicted by de Gennes and Pincus ${ }^{1}$ and verified experimentally by recent cryo-TEM (transmission electron microscopy) studies. ${ }^{2,3}$ The presence of an external magnetic field not only can induce the growth and alignment of chains but also introduces lateral interactions between these chains, resulting in the formation of elongated magnetic sheets. Simulations have predicted a variety of solid equilibrium phases in the presence of a magnetic field, among which the body-centered-tetragonal, face-centeredcubic (fcc), ${ }^{4}$ and hexagonal phases ${ }^{5,6}$ that can coexist with the chain phase. Here, we present new stroboscopic small angle neutron scattering (SANS) measurements to characterize three magnetic nanoparticle systems with different dipolar dynamics.

Previous static measurements with SANS including polarized neutrons (SANSPOL) and cryo-TEM in concentrated Co-FF (Refs. 7-9) and $\mathrm{Fe}_{3} \mathrm{O}_{4}$-FF (Refs. 3 and 10) revealed unusual hexagonal ordering of nanosized magnetic particles induced by an external magnetic field. Locally ordered hexagonal domains were found to coexist with flexible dipolar chains, the sizes and volume fractions of which depend on the strength of the applied field. ${ }^{8}$ The correlation length was estimated to be $60-100 \mathrm{~nm}$ from the width of the Bragg peaks.
To study the dynamics of the formation and disintegration of magnetic structures, we performed time-resolved SANS measurements. Dynamical investigations of nanosized inhomogeneities by means of SANS are usually limited to slow processes where during data acquisition time (typically of the order of few minutes) the system remains in a quasisteady state. The dynamical time is considerably reduced when such processes are repeated in a reproducible pathway and data collection can be synchronized with the periodic process. In a first type of experiment, the relaxation of the local ordering toward equilibrium has been studied when the saturation magnetic field was switched off. ${ }^{11}$ Magnetic correlations between Co nanoparticles were found to decay exponentially with a characteristic time of the order of $1-5 \mathrm{~s}$. The onset of the local ordering, however, was too fast to be observed by this direct mode. In a second type of experiment, the time-resolved SANS response to periodic perturbation from a sine-wave modulation of the external magnetic field was analyzed. Two techniques for investigations of oscillating processes by means of SANS have been compared: ${ }^{12}$ a direct stroboscopic mode, which uses a continuous neutron flux, and a new pulsed time-resolved technique, TISANE, proposed by Gähler and Golub, ${ }^{13}$ which allows the time resolution to be extended to submillisecond range.

Thermal reorientation of the magnetic moment in a magnetic colloid can take place either by Brownian rotation of 
TABLE I. Structural and magnetic parameters of samples.

\begin{tabular}{llll}
\hline \hline Sample & Co-FF $(\mathrm{MFT} 3)^{\mathrm{a}}$ & $\mathrm{Fe}_{3} \mathrm{O}_{4}$-FF $^{\mathrm{b}}$ & $\mathrm{CuCo}$ alloy \\
\hline Surfactant shell thickness $D(\mathrm{~nm})$ & $1.9 \pm 0.2$ & $2.1 \pm 0.2$ & 0 \\
Volume-weighted average core radius $R_{c}(\mathrm{~nm})$ & 4.4 & 9.0 & 4.8 \\
Standard deviation of the log-normal distribution & 0.13 & 0.20 & 0.15 \\
over $R_{c}$ & & & 7 \\
Magnetic particle moment $\mu\left(10^{19} \mathrm{~A} \mathrm{~m}^{2}\right)$ & 5.3 & 15.5 & \\
dipole-dipole attraction $E_{d d}(\max )\left(k_{B} T\right)$ & -4 & -9 & -6.72 \\
Interaction energy $E_{B}$ with $40 \mathrm{mT}$ at $300 \mathrm{~K}\left(k_{B} T\right)$ & -5.2 & -15.2 & 1.023 \\
Contrast ratio $\Delta \eta_{\text {nuc }} / \Delta \eta_{\text {mag }}$ & 0.29 & 5 & $\mathrm{Cu}$ \\
Solvent/matrix & $\mathrm{L} 9$ & $\mathrm{Decalin}$ & $\infty$ \\
Viscosity solvent $(\mathrm{Pa} \mathrm{s})$ & 0.2 & $2.5 \times 10^{-3}$ & $\infty$ \\
\hline \hline
\end{tabular}

Reference 11.

${ }^{\mathrm{b}}$ Reference 10 .

${ }^{\mathrm{c}}$ Reference 17.

the particle and/or by Néel relaxation of the particle moment. The first mechanism has a relaxation time $\tau_{B}$ that depends on the hydrodynamic radius $R_{h}$ and the viscosity $\eta$ of the solvent according to

$$
\tau_{B}=\frac{4 \pi \eta R_{h}^{3}}{k_{B} T},
$$

where $k_{B}$ is the Boltzmann constant and $T$ the absolute temperature. The Néel mechanism involves rotation of the magnetization vector inside a particle against an energy barrier $K_{a} V_{c}$ with a characteristic time $\tau_{N}$ given by

$$
\tau_{N}=\frac{1}{2 \pi f_{0}} \exp \left(\frac{K_{a} V_{c}}{k_{B} T}\right) .
$$

$f_{0}$ is a constant in the range $10^{7}-10^{12} \mathrm{~s}^{-1}, K_{a}$ is a shapedependent material constant, and $V_{c}$ is the volume of the magnetic core, equal to $V_{c}=(4 / 3) \pi R_{c}^{3}$ for a sphere with radius $R_{c}$. The apparent characteristic time is given by $\tau_{\text {app }}^{-1}$ $=\tau_{B}^{-1}+\tau_{N}^{-1}$, which means that the fastest mechanism dominates for individual particles.

In this study, we focus on the dynamics of three magnetic nanoparticle systems: two ferrofluids (FFs) studied in the low temperature range around the freezing point of the carrier liquid (where slowing down of the moment reorientation is expected), and for comparison, a dispersion of cobalt particles in metallic copper. In the case of the ferrofluids, dipolar interactions between particles influence both the Néel and the Brownian mechanisms either by formation of larger domains with correspondingly longer relaxation times $\tau_{B}$ or by modification of the energy barrier $K_{a} V_{c}$ in Eq. (2). For the latter case longer ${ }^{14}$ or shorter relaxation times have been predicted $^{15}$ and indications of collective phenomena have been reported. ${ }^{16}$ In the case of single-domain superparamagnetic Co precipitates embedded in a solid $\mathrm{Cu}$ matrix, magnetic particle moments can be reoriented only via the Néel mechanism. The temperature and frequency dependence should allow establishing the relevant relaxation mechanisms of nanoparticle moments and the influence of the dipolar interactions.

\section{EXPERIMENT}

Two types of ferrofluids with nearly monodisperse nanoparticles have been investigated, namely, concentrated Co ferrofluid ("MFT3") $8,11,12$ with a cobalt core of radius $R_{c}$ $=4.4 \mathrm{~nm}$ dispersed in a viscous synthetic hydrocarbon oil "Edwards L9" (monoalkylnaphthalene) and magnetite $\left(\mathrm{Fe}_{3} \mathrm{O}_{4}\right)$ particles of $R_{c}=9.0 \mathrm{~nm}$ dispersed in decalin,, 30 both stabilized by a surfactant layer of a thickness $D$ of about $2 \mathrm{~nm}$. The maximal dipolar interaction energy $E_{d d}(\max )$ for particles with saturation magnetization $M_{\text {sat }}$ in head-to-tail conformation and closest contact $\sigma=2\left(R_{c}+D\right)$ is given by

$$
E_{d d}(\max )=-\frac{\mu_{0}}{2 \pi} \frac{M_{s a t}^{2} V_{c}^{2}}{\sigma^{3}}
$$

and amounts to similar values for both ferrofluids. However, the viscosity of Co-FF system is by 2 orders of magnitudes higher than the $\mathrm{Fe}_{3} \mathrm{O}_{4}$ - $\mathrm{FF}$ (Table I). The results are compared to the dynamics of superparamagnetic Co precipitates of $R_{c}$ $=4.8 \mathrm{~nm}$ embedded in a solid $\mathrm{Cu}$ matrix. ${ }^{17}$

A magnetic field generating coil was built around a ferrite core and equipped with a high-stability frequency generator and power supply. Periodic sine-wave modulations of the horizontal magnetic field up to amplitudes of $B_{0}=40 \mathrm{mT}$ and frequencies between $\nu_{S}=50$ and $500 \mathrm{~Hz}$ have been applied to the sample placed in the homogenous part of the magnetic field. Optionally, a static field up to $B_{\mathrm{st}}=20 \mathrm{mT}$ could be superimposed. The reversibility of the reordering process in the dynamic mode has been checked by comparing the stroboscopic SANS results with those measured in the same coil device powered by a dc that produces a static magnetic field of the same amplitude. When $m_{0}$ is the spontaneous magnetization the interaction energy of the particle moment, $E_{B}$ $=V_{c} m_{0} B(t)$ exceeds the thermal energy $k_{B} T$ even at $300 \mathrm{~K}$ and hence should produce significant alignment along $B$. An automatic temperature control unit between 300 and $66 \mathrm{~K}$ was achieved by using a Stirling-type closed-cycle refrigerator $(\mathrm{CCR})$ where the sample was mounted onto the cold finger of the CCR and, with the surrounding aluminium 


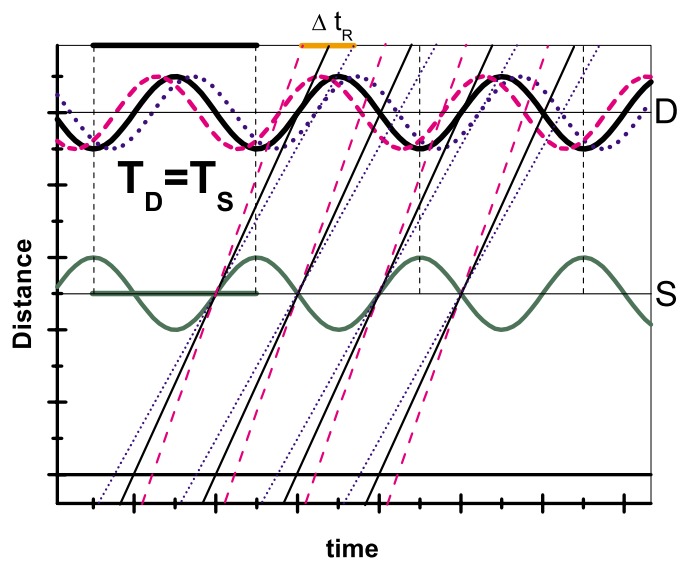

FIG. 1. (Color online) Distance versus time diagram of the continuous stroboscopic SANS technique. Neutrons of a fixed wavelength (solid lines) are scattered at the sample (S) in the oscillation state defined by the frequency $\nu_{S}$. Due to the flight time, they are recorded in the detector (D) with some delay. When the detector acquisition frequency $\nu_{D}$ is synchronized with $\nu_{S}$, the time-resolved response (solid curve) is obtained with good statistics after a sufficient number of cycles. Due to the wavelength distribution of the incident neutrons, the response of faster (dashed pink lines) or slower neutrons (dotted blue lines) appears earlier (dashed pink curve) or later (dotted blue curve). The superposition of contributions at different wavelengths gives rise to a damping of the observed oscillations that increases with the frequency of the applied field.

vacuum can, was adjusted in the middle of a $30 \mathrm{~mm}$ gap of the ferrite yoke.

Stroboscopic experiments have been performed on the SANS instruments V4 at BENSC, Berlin, and D22 at ILL, Grenoble, using a continuous neutron beam with a wavelength band of $\Delta \lambda / \lambda=0.11$ at $\lambda=0.608 \mathrm{~nm}$. The area detector was placed at a distance of $L_{\mathrm{SD}}=4 \mathrm{~m}$ from the sample. Data acquisition was triggered by the frequency generator at zero ac voltage producing histograms of $128 \times 128$ pixels of $0.5 \times 0.5 \mathrm{~cm}^{2}(\mathrm{~V} 4)$ and $0.8 \times 0.8 \mathrm{~cm}^{2}$ (D22) for position and $n=100$ time channels of widths $\Delta t=\left(n \nu_{S}\right)^{-1}$.

Neutrons of a given wavelength hit the sample at a given phase of the ac magnetic field. The scattered neutrons arrive at the detector after a flight time $t_{\text {TOF }}$ given by

$$
t_{\mathrm{TOF}}[\mathrm{s}]=\lambda[\mathrm{nm}] L_{S D}[\mathrm{~m}] 2.52778 \times 10^{-3},
$$

which leads to a delay of the response in the detector by $t_{\mathrm{TOF}} / \Delta t$ channels with respect to the magnetic field.

Figure 1 illustrates the limitation of this technique, which results from the instrumental wavelength distribution of $\Delta \lambda / \lambda=0.11$, which gives rise to the spread of $t_{\mathrm{TOF}}$ time. The response from the faster neutrons is recorded earlier in the detector than that of the slower ones, which leads to a superposition of intensities oscillating with the same frequency but with different phases. Using the notation $T_{S}=\nu_{S}^{-1}$ for the oscillation period of the magnetic field, the relative time spread $\varepsilon=\Delta t_{R} / T_{S}$ of the signal increases linearly with the frequency according to

$$
\varepsilon=\frac{\Delta \lambda}{\lambda} t_{\mathrm{TOF}} \nu_{S}
$$

which results in a smearing of the oscillations in the detector response. For the present case of the triangular wavelength distribution of $\Delta \lambda / \lambda=0.11$ produced by the velocity selector, $\lambda=0.608 \mathrm{~nm}$ and at $L_{\mathrm{SD}}=4 \mathrm{~m}$, Eq. (5) yields $\Delta t_{R}=0.68 \mathrm{~ms}$. At $\nu_{S}=600 \mathrm{~Hz}$, the full period of the magnetic field is $T_{S}$ $=1.66 \mathrm{~ms}$, which is already very close to the spread of neutron arrival times. We note that for higher frequencies and/or larger distances, the time resolution of the continuous stroboscopic technique is no longer sufficient and must be replaced by the pulsed TISANE technique. ${ }^{12}$ In the present continuous study, we have not exceeded a frequency $\nu_{S}$ of $400 \mathrm{~Hz}$. Intensities have been corrected pixel by pixel for background, transmission, and detector efficiency and normalized to the scattering of a water standard using the software BERSANS. The $Q$ range $0.2 \mathrm{~nm}^{-1}<Q<1.2 \mathrm{~nm}^{-1}$ and resolution of the order of $\Delta Q / Q<0.1$ allowed us to resolve properly the characteristic correlation peaks of the local hexagonal ordering that appear for the Co-FF samples at $Q_{1}$ $=0.4 \mathrm{~nm}^{-1}$ and $Q_{2}=0.3 \mathrm{~nm}^{-1}, 8,9$ and for $\mathrm{Fe}_{3} \mathrm{O}_{4}$ at $Q_{1}$ $=0.36 \mathrm{~nm}^{-1}$ and $Q_{2}=0.34 \mathrm{~nm}^{-1} \cdot{ }^{10}$ In order to analyze the full time dependence, intensities have been integrated in angle sectors over a width of $20^{\circ}$ at angles $\alpha=0^{\circ}, 30^{\circ}, 60^{\circ}$, and $90^{\circ}$. Data analysis by simultaneous fits was performed using the software SASFIT.

ac susceptibility measurements have been performed on both ferrofluids in a frequency range between $0.1 \mathrm{~Hz}$ and $100 \mathrm{kHz}$ using a differential transformer device as described in Ref. 18.

\section{THEORY}

The SANS intensity for single-domain monodisperse magnetic particles at a scattering vector $Q$ is given by

$$
\begin{aligned}
I(\mathbf{Q}, \alpha, B, T)= & f_{e} \int_{\lambda-\Delta \lambda}^{\lambda+\Delta \lambda}\left(\left(F_{M}^{2} L^{2}(x) \sin ^{2} \alpha+F_{N}^{2}\right) S(\mathbf{Q}, \alpha, t)\right. \\
& +F_{M}^{2}\left\{2 L(x) / x-\sin ^{2} \alpha\left[L^{2}(x)-1\right.\right. \\
& +3 L(x) / x]\}) p(\lambda, \Delta \lambda) d \lambda+\left(1-f_{e}\right) U\left(\mathbf{Q}, \alpha, \nu_{s}\right),
\end{aligned}
$$

where $F_{N}$ and $F_{M}$ are the nuclear $(N)$ and magnetic $(M)$ form factors of the particles of radius $R$ defined by $F_{N, M}(Q R)$ $=\int d^{R} 4 \pi r^{2} \Delta \eta_{N, M}(\sin Q R / Q R)$ with the corresponding scattering contrasts $\Delta \eta_{N, M}=\eta_{N, M}-\eta_{\text {matrix }} . \alpha$ is the angle between $Q$ and the applied magnetic field $B . S(Q, \alpha, t)$ is an effective dynamical structure factor, which accounts for anisotropic interparticle interactions. The integral term in Eq. (6) was obtained for single-domain particles in equilibrium. ${ }^{19,20}$ The time dependence of the magnetic field enters in Eq. (6) via the Langevin function

$$
L(x)=\operatorname{coth}(x)-\frac{1}{x} \quad \text { where } x=\frac{V_{c} m_{0} B(t)}{k_{B} T} .
$$

As long as all particle moments follow the oscillating magnetic field, the SANS pattern should be described by Eq. (6) using 


$$
B(t)=B_{0} \sin \left[2 \pi \nu_{S}\left(t+t_{\mathrm{TOF}}\right)+\varphi\right]+B_{s t},
$$

where $B_{0}$ and $\varphi$ are the amplitude and an arbitrary phase of the oscillating field, respectively, and $B_{\mathrm{st}}$ is the superimposed static field. The term in braces is convoluted with $p(\lambda, \Delta \lambda)$, which describe the triangular wavelength distribution to account for the smearing of the oscillations. The factor $f_{e}$ gives the fraction of superparamagnetic moments that can follow the oscillating field, whereas $\left(1-f_{e}\right)$ is the fraction of moments that cannot follow and leads to a time-independent scattering contribution $\left(1-f_{e}\right) U\left(Q, \alpha, \nu_{S}\right)$. We assume that all particles that cannot follow the ac field are part of aggregates, the Brownian rotation of which gives very long relaxation times.

The important question is whether the effective dynamical structure factor $S(Q, \alpha, t)$ in Eq. (6) will depend on the applied magnetic field. If not, the intensity will depend on the magnetic field only via the Langevin parameter $x$ according to Eqs. (7) and (8). In the direction along the magnetic field, for $\alpha=0^{\circ}$, the integral term of Eq. (6) simplifies to $F_{N}^{2} S(Q, \alpha=0, t)+F_{M}^{2} 2 L(x) / x$, which yields a maximum at $B$ $x \rightarrow 0$

$=0$, since $2 L(x) / x \rightarrow 2 / 3$ while for finite magnetic fields this term is smaller than $2 / 3$ and vanishes for full alignment of the particle moments. For all other directions, additional magnetic terms add up in such a way that at $B=0$ the intensity $I(\alpha \neq 0)$ will always be lower than in the maximum field. This means that the intensities for $\alpha=0^{\circ}$ and $\alpha \neq 0^{\circ}$ are expected to oscillate in an opposite way as a function of time. Conversely, in-phase oscillating intensities for all angles can only be observed when the structure factor $S(Q, \alpha, t)$ itself depends on the magnetic field. It that case, the term $F_{N}^{2} S(Q, \alpha, t)$ for $\alpha=0^{\circ}$ increases with $B$ which leads to an intensity maximum at $B_{\max }$ and minimum at $B=0$. Note that only in the case where $F_{N}^{2} S(Q, \alpha, t)$ is the dominant term $\left[F_{N}^{2} S(Q, \alpha, t)>(2 / 3) F_{M}^{2}\right]$ the time-dependent structure factor can be extracted reliably from the oscillating intensities. This condition is clearly fulfilled in the $\mathrm{Fe}_{3} \mathrm{O}_{4}$-FF samples, for which $\Delta \eta_{\text {nuc }} / \Delta \eta_{\text {mag }} \approx 5$. This implies that for all angles, the scattering intensity follows the oscillating field, as is depicted in Fig. 2. For Co-FF, where $\Delta \eta_{\text {nuc }} / \Delta \eta_{\text {mag }} \approx 0.28$, antiphase oscillations are expected.

\section{RESULTS}

Both ferrofluid samples have been cooled down from 300 to $100 \mathrm{~K}$ either in zero magnetic field [zero-field cooling (ZFC)] or by applying a static field $B_{\text {st }}$ of $20 \mathrm{mT}$ [field cooling (FC)]. Starting from $100 \mathrm{~K}$, SANS measurements have been performed at each temperature first in a static field of $B_{\mathrm{st}}=0,10$, and $20 \mathrm{mT}$, then in an oscillating field at frequencies of 100,200 , and $300 \mathrm{~Hz}$, where the amplitude of the ac field was $B_{0}= \pm 10$ and $\pm 20 \mathrm{mT}$ and finally by superimposing a static field of $B_{\text {st }}=10$ and $20 \mathrm{mT}$.

\section{A. Magnetite ferrofluid}

\section{Room-temperature dynamics}

Figure 3 shows SANS scattering patterns corresponding to three different phases of the magnetic field (time channels)

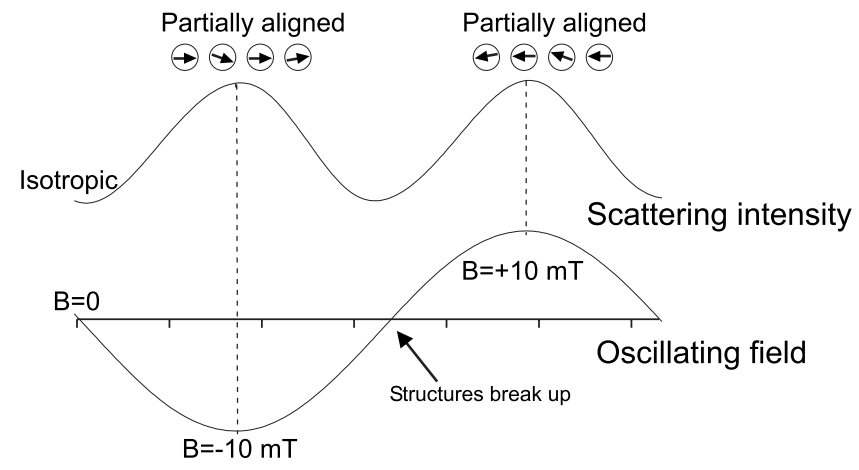

FIG. 2. Schematic illustration of the scattering intensity of a ferrofluid as a function of the applied oscillating magnetic field. At $B=0$, the scattering pattern is nearly isotropic (assuming immediate break up of the structures), while at $B= \pm 10 \mathrm{mT}$ the magnetic moments become aligned, which leads to anisotropic scattering patterns. Depending on the actual values of magnetic and nuclear contrasts, the intensities $I(Q \| B)$ and $I(Q \perp B)$ are expected to oscillate in antiphase (Co-FF, see Fig. 8), while for $\mathrm{Fe}_{3} \mathrm{O}_{4}$-FF the field dependent $S(Q, \alpha, t)$ yields in-phase oscillations (see Fig. 4).

for $\nu_{S}=100 \mathrm{~Hz}$ (panels a, b, and c) when an alternative magnetic field is applied with amplitude $B_{0}= \pm 10 \mathrm{mT}\left(B_{\mathrm{st}}=0\right)$. At this frequency, the oscillations due to the oscillating magnetic field are clearly visible in the two-dimensional scattering patterns. For the time channel that exactly corresponds to $B_{0}$ [see Fig. 3(c) for $B=-10 \mathrm{mT}$ ], the scattering intensity is anisotropic, while for $B=0$ [see Fig. 3(a)] the pattern is approximately isotropic. In the static case of $B_{\mathrm{st}}=10 \mathrm{mT}$ [Fig. $3(\mathrm{~d})$ ], the scattering pattern indicates the maximum anisotropy that can be obtained at this field strength. At this static field strength, the formed chains and sheets partially align along the external field. In the dynamic experiment, the

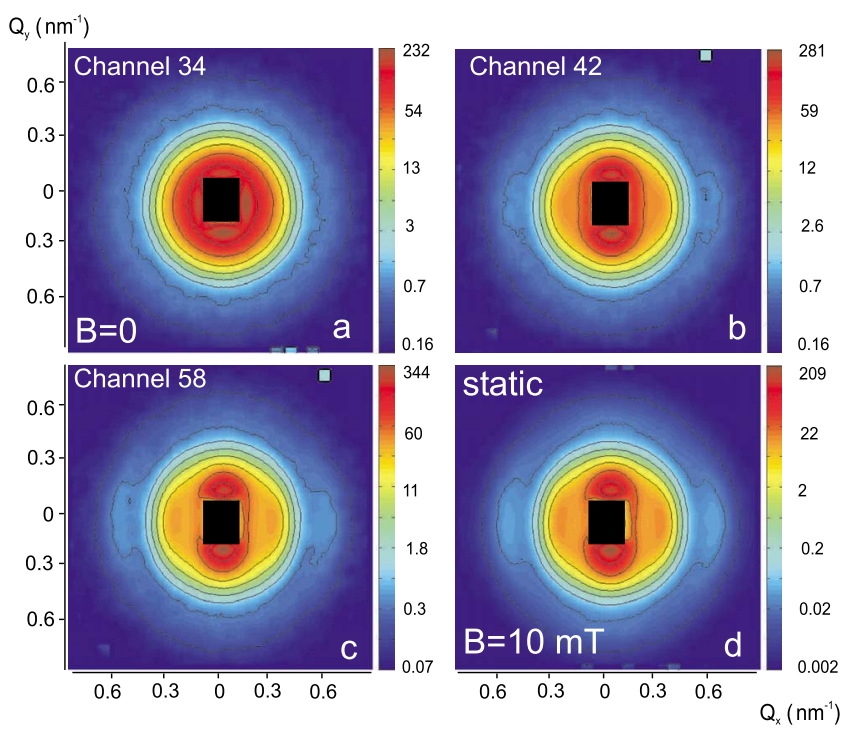

FIG. 3. (Color online) Room-temperature SANS patterns of $\mathrm{Fe}_{3} \mathrm{O}_{4}$-FF at $100 \mathrm{~Hz}$ and $B_{0}= \pm 10 \mathrm{mT}$, at time channels (a) 34 $(B=0)$, (b) 42 (intermediate B), and (c) $58\left(B=B_{0}\right)$. Panel (d) shows the corresponding static scattering pattern for $B_{\mathrm{st}}=10 \mathrm{mT}$. The horizontal magnetic field was aligned perpendicular the incoming neutron beam. Intensities are in arbitrary units. 

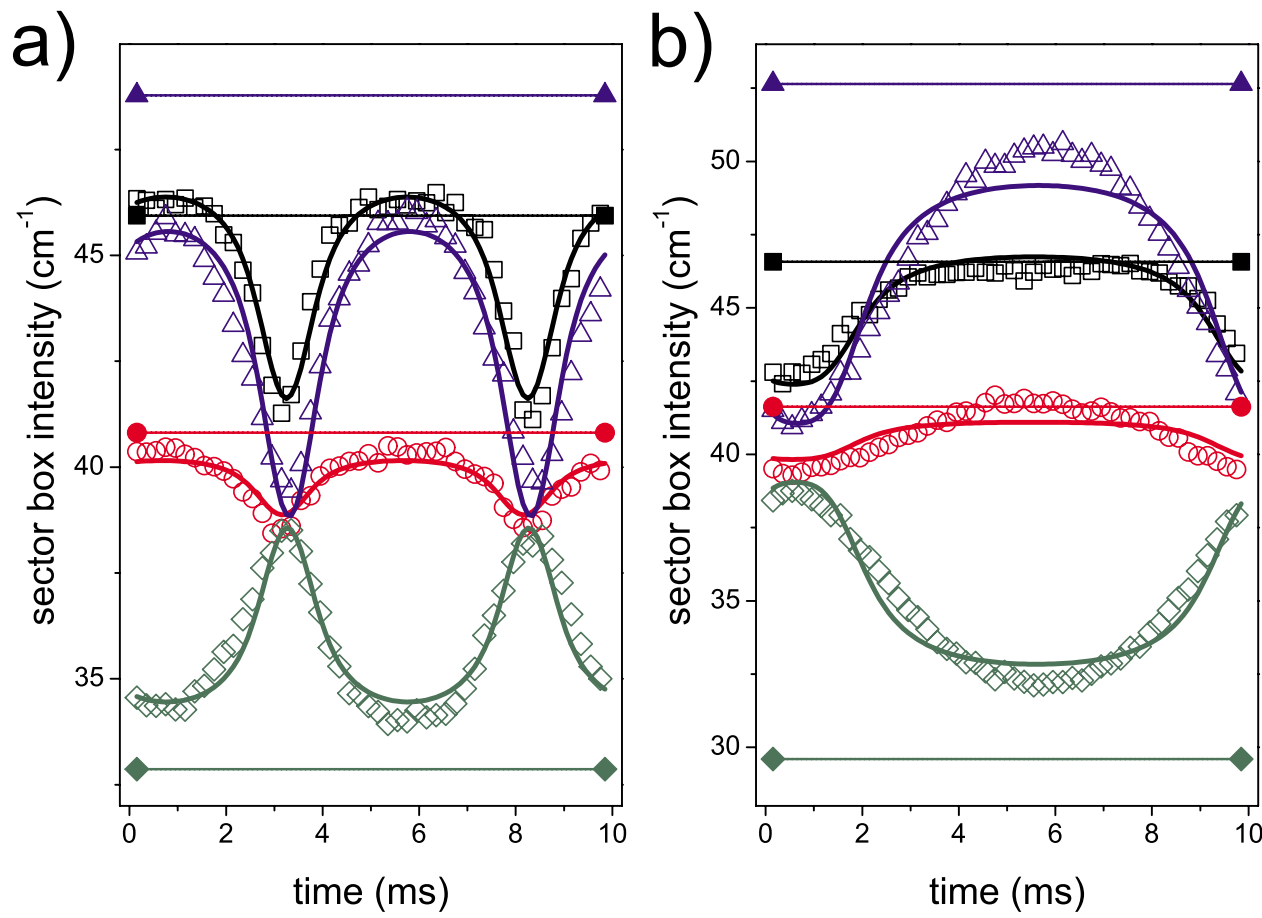

FIG. 4. (Color online) Time dependence of $\mathrm{Fe}_{3} \mathrm{O}_{4}$-FF around $Q_{1}$ and $Q_{2}$ at $B_{0}=20 \mathrm{mT}, T=296 \mathrm{~K}$. The open symbols are the experiments at $100 \mathrm{~Hz}$ (for the sake of clarity, only one out of two measured points is shown); the solid lines are a fit to the data using Eq. (6) and the solid symbols are the static measurements for the different angles. Square: $0^{\circ}$, circle: $30^{\circ}$, diamond: $60^{\circ}$, and triangle up: $90^{\circ}$. (a) $B_{\text {st }}=0$; (b) with an additional static field of $B_{\mathrm{st}}=20 \mathrm{mT}$.

steady state structures only partially form and the steady state hexagonal SANS pattern is only partially established. The full time dependence of the integrated intensities $I\left(Q_{i}, \alpha\right)$ for $\nu=100 \mathrm{~Hz}$ in the $Q$ range $0.28-0.38 \mathrm{~nm}^{-1}$, using sector boxes averaged over a width of $20^{\circ}$, is shown in Fig. 4(a) for $\alpha=0^{\circ}, 30^{\circ}, 60^{\circ}$, and $90^{\circ}$.

Note that this is the $Q$ range in which the peaks are expected when hexagonal sheets are formed. ${ }^{10}$ In addition, the corresponding values for the static case are presented in Fig. 4(a) (horizontal lines). The periodicity of the response is twice that of the magnetic field. For all directions except $\alpha$ $=60^{\circ}$, the oscillations of the intensities are in phase with the applied magnetic field, which clearly shows a strong contribution from a time-dependent (i.e., field-dependent) structure factor. The intensities in the directions of $\alpha=0^{\circ}$ and $\alpha=30^{\circ}$ come closer to the steady state value than in the directions $\alpha=60^{\circ}$ and $\alpha=90^{\circ}$. The observed response suggests a time dependence of $S(Q, \alpha, t)$ that must be related to the magnitude of the magnetic field. The best agreement of the observed intensities was obtained (see solid lines in Fig. 4) for all angles $\alpha$ with the model function for the dynamical structure factors given by

$$
S(Q, \alpha, t)=1+[S(Q, \alpha)-1] L^{2}(x),
$$

where $S(Q, \alpha)$ gives the time-averaged static structure factor, which result from correlations between neighboring particles. At zero field, particle moments are randomly oriented and therefore the dynamical structure factor is equal to $S(Q, \alpha, t)=1$. However, as soon as the magnetic field is applied, the dynamical structure factor $S(Q, \alpha, t)$ can be larger (for $\alpha=0^{\circ}, 30^{\circ}$, and $90^{\circ}$ ) or smaller (for $\alpha=60^{\circ}$ ) than $1 \mathrm{de}-$ pending on the actual value of $S(Q, \alpha)$.

The formation of field-induced structures is mainly governed by the dipole-dipole coupling, which scales with $\mu^{2}$. Therefore, the dynamical structure factor $S(Q, \alpha, t)$ depends on $L^{2}(x)$ in this model. The experimental data in Fig. 4 have been simultaneously fitted using Eqs. (6)-(9), with the constraints of common parameters from Table I. As a result, the values for the dynamical structure factors $S(Q, \alpha, t)$ can be determined for each temperature. As shown in Fig. 4, excellent agreement was obtained when using an identical set of global parameters for both the dynamic data and the static data. Uncertainties on fitted parameters depend mainly on the amplitude of oscillation, and were evaluated to approximately $+/-10 \%$. At $\nu=100 \mathrm{~Hz}$ and $B_{0}= \pm 10 \mathrm{mT}$, the fraction of particles that can follow the oscillating field is about $80 \%$. The other fraction $(20 \%)$ gives rise to a static background contribution: particles aggregate into domains too large to follow the oscillating field. In the frequency range $160-300 \mathrm{~Hz}$, the parameters $f_{e}, S(Q, \alpha, t)$, and $U$ hardly change.

\section{Zero-field cooling}

When the $\mathrm{Fe}_{3} \mathrm{O}_{4}$-FF had been cooled down in zero field to $150 \mathrm{~K}$, the scattering patterns (not shown) remained nearly isotropic and identical when either measured in zero field, or in a static field of $20 \mathrm{mT}$ [corresponding to $x=16$ in Eq. (7)], an oscillating magnetic field of $20 \mathrm{mT}$, or even when a dc field of $20 \mathrm{mT}$ is superimposed. This implies that practically no reorientation of the magnetic moments is achieved in 


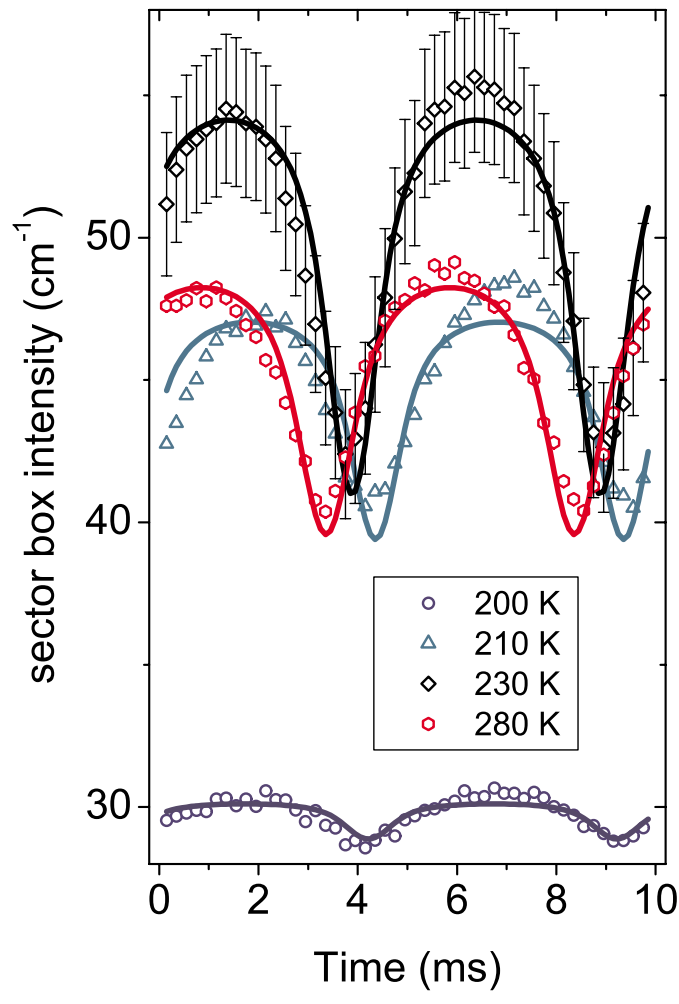

FIG. 5. (Color online) The sector box intensity of $\mathrm{Fe}_{3} \mathrm{O}_{4}$-FF as a function of the time for a frequency of $100 \mathrm{~Hz}$ at different temperatures for $\alpha=90^{\circ}$ direction $\left(B_{0}= \pm 20 \mathrm{mT}\right)$, upon heating after ZFC (for the sake of clarity, only one out of two measured points is shown). Solid lines correspond to the theoretical model, which result from the simultaneous fit of static and dynamic data at $0^{\circ}, 30^{\circ}$, $60^{\circ}$, and $90^{\circ}$. Error bars are shown for values at $230 \mathrm{~K}$.

static or dynamic fields of $20 \mathrm{mT}$. In the frozen state at $150 \mathrm{~K}$, Brownian rotation is impeded and even Néel relaxation does not play a significant role since no measurable effect of the applied magnetic fields has been detected. Therefore, the randomly oriented structures at $150 \mathrm{~K}$ can be considered as completely arrested (in agreement with Ref. $10)$.

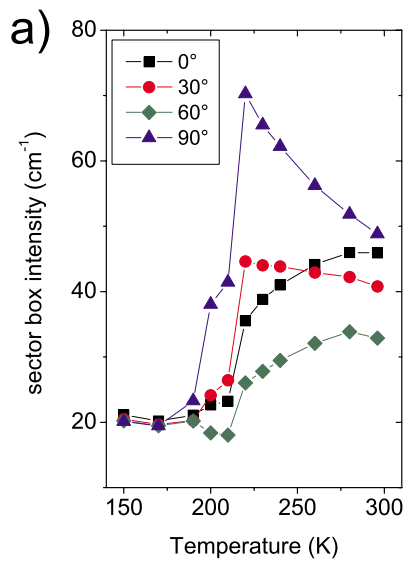

Next, we applied an oscillating magnetic field of $20 \mathrm{mT}$ and increased the temperature. Figure 5 depicts the sector box intensities versus the time for $90^{\circ}$ as a function of the temperature. At low temperature $(200 \mathrm{~K})$, hardly any oscillations are present as a result of the solid matrix, and it is only at $210 \mathrm{~K}$ that the oscillations start to be clearly visible. Note that with decreasing temperature, the oscillations feature a small phase shift, indicating the retarded formation dynamics of the structures at lower temperature.

To better highlight the melting of the ferrofluid (after zero-field cooling), the sector box intensities in the $Q$ range $0.28-0.38 \mathrm{~nm}^{-1}$ are presented as a function of the temperature when exposed to a static field of $20 \mathrm{mT}$ [Fig. 6(a)]. The strong increase of the intensities at $T_{f}=210 \mathrm{~K}$ is assigned to the melting temperature and results from alignment of the chains and/or from unblocking of the individual moments. Above the melting point, the scattering intensity in the $90^{\circ}$ direction drops with increasing temperature due to the breaking up of dipolar structures.

The structure factor $S(Q, \alpha)$ shows a similar temperature dependency as the sector box intensities, as is depicted in Fig. 6(b). The values of $S(Q, \alpha)$ were determined from dynamic experiments at $B_{0}= \pm 20 \mathrm{mT}$ by simultaneously fitting the scattering intensities in all directions together with the corresponding data measured in a static field as an internal reference. At room temperature, $S(Q)$ in the $0^{\circ}$ direction is relatively high and reflects the residual intensity that is ascribed to the first-order diffraction peak for single chains.

This suggests that single chains are the dominant structures at room temperature. The value of $S(Q)$ gradually decreases in this direction with decreasing temperature, indicative for the dissipation of single chains. In contrast, the intensities in the $30^{\circ}$ and $90^{\circ}$ directions increase until the melting point is reached. These trends suggest that there is a gradual transition from hexagons to chains when the temperature increases. Finally, the fraction $f_{e}$ was determined at different temperatures [Fig. 6(c)], indicating that the number of particles that can follow the field oscillations is negligible at low temperature and increases continuously with the temperature above $T_{f}$.
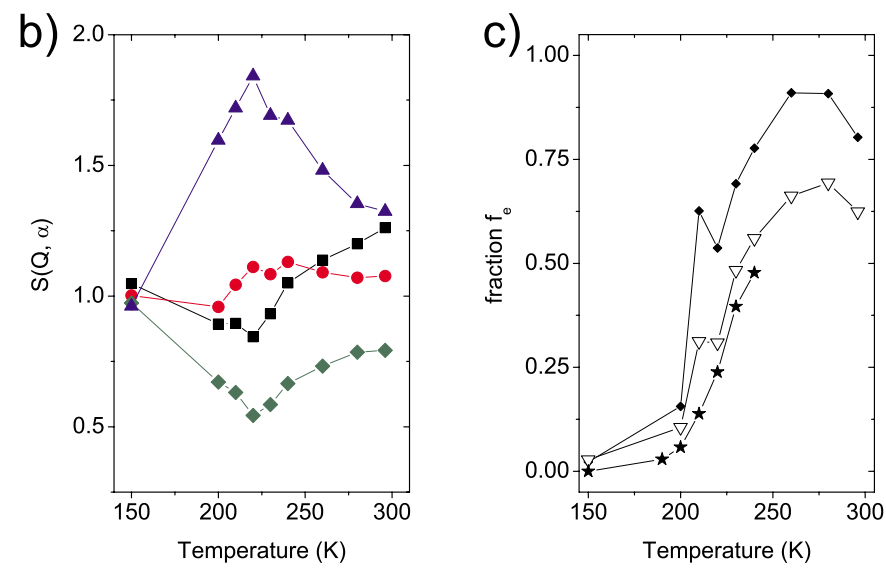

FIG. 6. (Color online) $\mathrm{Fe}_{3} \mathrm{O}_{4}$-FF: temperature dependence of (a) the sector box intensities for the four different angles in a static field of $B_{\mathrm{st}}=20 \mathrm{mT}$, (b) the structure factor $S(Q, \alpha)$ for $\left(B_{0}= \pm 20 \mathrm{mT}\right)$, and (c) the fraction $f_{e}$ of mobile moments at $100 \mathrm{~Hz}$ after ZFC at $B_{\mathrm{st}}=0$ (diamonds), after ZFC at $B_{\mathrm{st}}=20 \mathrm{mT}$ (triangles down), and after field cooling and measuring at $B_{\mathrm{st}}=20 \mathrm{mT}$ (stars). Errors are approximately within $10 \%$. 
Superposition of a static magnetic field. When a static field of $B_{\mathrm{st}}=20 \mathrm{mT}$ is superimposed to the ac field of $B_{0}$ $=20 \mathrm{mT}$, the time-dependent patterns now oscillate between $B=0$ (which is observed at $t=1 \mathrm{~ms}$ ) and $B_{0}=40 \mathrm{mT}$ (at $t$ $=6 \mathrm{~ms}$ ) with the frequency of the inducing field [Fig. 4(b)]. All curves are still well described by the same model of Eq. (6) when the static field $B_{\mathrm{st}}$ is taken into account. The fitted parameters are very close to the values obtained for $B_{\mathrm{st}}=0$ except the fraction $f_{e}$ of freely rotating moments and the constant $U$. The evolution of the fraction $f_{e}$ of mobile moments, plotted in Fig. 6(c) shows the same temperature dependence as for $B_{\mathrm{st}}=0$; however, the actual values of $f_{e}$ are always lower, e.g., at $280 \mathrm{~K} f_{e}\left(B_{\mathrm{st}}=20 \mathrm{mT}\right)=0.71$ instead of $f_{e}\left(B_{\mathrm{st}}=0\right)=0.89$. Correspondingly, the time-independent scattering contribution $\left(1-f_{e}\right) U(\alpha, \nu)$ from the arrested moments $U$ is higher than for $B_{\mathrm{st}}=0$. This suggests that the additional static field induces larger ordered structures with longer relaxation times.

We remark that by variation of the static field $B_{\text {st }}$ between 0 and $B_{0}$ the shape of the intensity versus time curves (not shown) changes tremendously between the extreme types shown in Fig. 4(a) (frequency-doubled response at low $B_{\text {st }}$ ) and Fig. 4(b) (same-frequency response at high $B_{\mathrm{st}}$ ). This kind of contrast variation technique can be used to probe the field dependence of the dynamical behavior of the systems.

\section{Cooling in a static magnetic field}

When the sample was cooled down (slowly) to $150 \mathrm{~K}$ in a static field of $B_{\mathrm{st}}=20 \mathrm{mT}$, the field-induced structures formed just above $T_{f}$ and aligned along the field remained fully arrested once an oscillating field was applied. Only upon heating above $T_{f}$, oscillations occur, which are again perfectly described by the previous model. The only differences occur in the parameters of $f_{e}$ [Fig. 6(c)] and $\left(1-f_{e}\right) U(\alpha, \nu)$, which is in line with an enhanced amount of larger structures where the moments cannot follow up the oscillating magnetic field.

\section{Relaxation on longer time scales}

In another setup, the time transient dynamics has been studied by switching the static magnetic field between 0 and $20 \mathrm{mT}$ with a frequency of $0.05 \mathrm{~Hz}$. The detailed results are presented elsewhere. ${ }^{21}$ Globally, we observed a superposition of an instantaneous process and a slow one. The latter is described by an exponential growth and decay with a characteristic time of the order of seconds. This slow relaxation process is at the origin of the time-independent scattering contribution in the data above.

\section{B. Cobalt ferrofluid}

\section{Cooling in zero magnetic field}

During ZFC, the total intensity of the overall isotropic scattering patterns showed a steep decrease at $T_{f}=119 \mathrm{~K}$, which reflects the freezing of the system in zero field. The scattering pattern at $T=100 \mathrm{~K}$ and zero field was isotropic and identical to the room-temperature pattern at $B=0$. When a static field of $20 \mathrm{mT}$ is applied, the patterns remain almost isotropic, i.e., once frozen realignment of the structures is
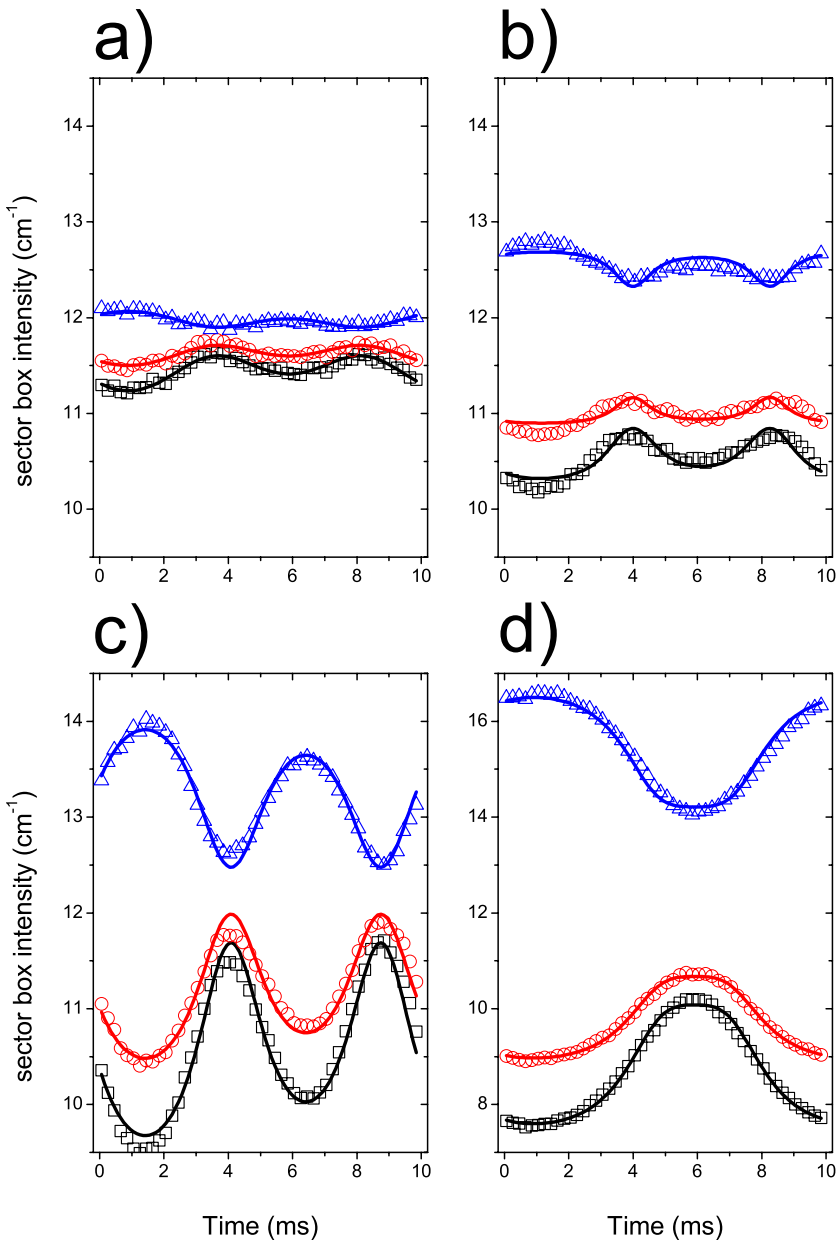

FIG. 7. (Color online) ZFC, sector box intensities at $\alpha=0^{\circ}, 30^{\circ}$, and $90^{\circ}$ of Co-FF in ac field of $100 \mathrm{~Hz}$ at $B_{0}=20 \mathrm{mT}$ and (a) 100 (b) 150 , (c) 300 , and (d) $300 \mathrm{~K}$ with an additional static field $B_{\text {st }}$ $=20 \mathrm{mT}$. For the sake of clarity, only one out of two measured points is shown, and static values are not shown but were included in the fit.

almost suppressed. With increasing temperature, the scattering patterns are more and more anisotropic, which results from a continuous alignment of moments along the external static field. Above $180 \mathrm{~K}$, no further evolution was observed in a static field of a given strength, i.e., the system was in thermal equilibrium.

Applying an oscillating field at $100 \mathrm{~K}$, the intensity slightly oscillates as a function of time. The intensities integrated over angle sector boxes of $20^{\circ}$ in widths and in the $Q$ range between 0.2 and $0.45 \mathrm{~nm}^{-1}$ at the angles $\alpha=0^{\circ}, 30^{\circ}$, and $90^{\circ}$ (i.e., the $Q$ range corresponding to the correlation peaks) are compared in Fig. 7 to those of the corresponding static measurements: Even below $T_{f}$, at $100 \mathrm{~K}$ [Fig. 7(a)] some of the particle moments follow the oscillating field.

Simultaneous least-square fits in terms of Eq. (6) have been performed as before on all static and dynamic data measured at a given temperature for different orientations $\alpha$. Note that a weak residual remanent field of the order of $2 \mathrm{mT}$ had to be included to the fit to account for the observed asymmetry of the oscillations in Figs. 7 and 10. 

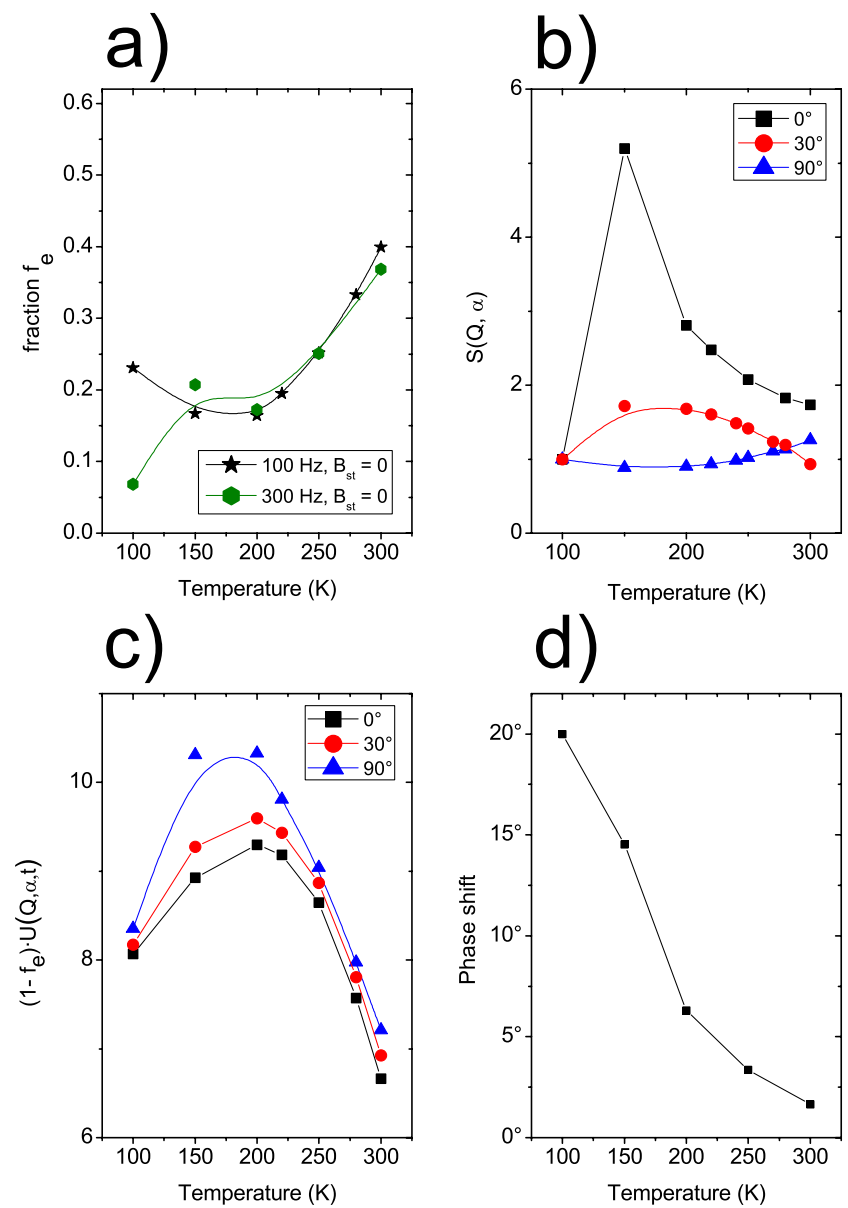

FIG. 8. (Color online) Temperature variation of the model parameters in Co-FF for ZFC data at $100 \mathrm{~Hz}$ and $B_{0}=20 \mathrm{mT}$ at $B_{\text {st }}$ $=0$ : (a) volume fraction $f_{e}$ of oscillating moments, (b) field-induced structure factors $S\left(Q, \alpha=0^{\circ}, 30^{\circ}, 90^{\circ}\right)$, (c) static contribution (1 $\left.-f_{e}\right) U\left(\alpha=0^{\circ}, 30^{\circ}, 90^{\circ}\right)$, and (d) phase shift extrapolated to $\nu=0$.

In the Co-FF sample, where the magnetic contrast is dominating, the scattering intensities at $\alpha=0^{\circ}$ and $\alpha=90^{\circ}$ are not very sensitive to structure factors. In fact, the dynamic part could have been fitted with a set of parameters using time-independent structure factors. However, at lower temperatures, the values of $S(Q)$ would then be different in a static or oscillating field of same strengths. Instead, we had again to assume dynamical structure factors $S(Q, \alpha, t)$ depending on the magnetic field according to Eq. (9).

The temperature evolution of the model parameters is shown in Fig. 8 for $\nu=100 \mathrm{~Hz}$ and $B_{0}=20 \mathrm{mT}$.

At $100 \mathrm{~K}$, the static contributions $\left(1-f_{e}\right) U(\alpha, \nu)$ for $\alpha$ $=0^{\circ}, 30^{\circ}$, and $90^{\circ}$ are nearly identical (Fig. 8(c)) and correspond to those observed in zero field at $250 \mathrm{~K}$. In the frozen state, the oscillating part could not be analyzed by using the value of a particle moment corresponding to the average volume of the Co core. Instead, the argument $x$ in Eq. (7) was adjusted as a free parameter leading to a magnetic particle moment, which was 1 order of magnitude smaller. This indicates that at $100 \mathrm{~K}$ only particles with an average core radius of $2.4 \mathrm{~nm}$ can still follow the oscillating magnetic field. Despite the narrow size distribution, a fraction of smaller particles is always present for which the Néel relaxation is the dominating effect. Between 100 and $150 \mathrm{~K}$, the static contributions $\left(1-f_{e}\right) U(\alpha, \nu)$ depend more and more on the angle $\alpha$. The dominance of the contribution at $90^{\circ}$ is characteristic for a preferred orientation of chain segments along the field. The temperature dependency of $S(Q, \alpha)$ again demonstrates the dynamical nature of the local ordering: The value of $S\left(Q, \alpha=0^{\circ}\right)$ for the direction parallel to $B_{0}$ is very large at $150 \mathrm{~K}$ and decreases with increasing temperature while $S\left(Q, \alpha=30^{\circ}\right)$ and $S\left(Q, \alpha=90^{\circ}\right)$ remain near unity. This indicates that above $T_{f}$, an increasing amount of particles are involved in interparticle correlations forming segments of chains rather than hexagonal layers. The fraction of freely oscillating particle moments $f_{e}$ continuously increases from about $20 \%$ at $100 \mathrm{~K}$ (at $100 \mathrm{~Hz}$ ) to $40 \%$ at $300 \mathrm{~K}$ while the static contribution $U\left(\alpha=90^{\circ}\right)$ decreases.

This is in agreement with previous static data, which have shown a dominance of chainlike structures at low magnetic fields. ${ }^{8}$ In the temperature range between 150 and $300 \mathrm{~K}$, no significant frequency dependence of the parameters $f_{e}$, (1 $\left.-f_{e}\right) U(\alpha, \nu)$, and $S(Q, \alpha)$ has been detected. Only at $100 \mathrm{~K}$, the factor $f_{e}$ was found to decrease from $23 \%$ at $100 \mathrm{~Hz}$ to $8 \%$ at $300 \mathrm{~Hz}$ while $U\left(90^{\circ}\right)$ is increased. This indicates that the characteristic frequency for the single particle rotation at $100 \mathrm{~K}$ must be close to $300 \mathrm{~Hz}$ and corroborates the results of a previous TISANE experiment ${ }^{12}$ where the observed threshold frequency around $\nu=1300 \mathrm{~Hz}$ at $300 \mathrm{~K}$ was assigned to Brownian rotation of single-domain particles $\nu_{B}$ $=\left(2 \pi \tau_{B}\right)^{-1}$ where $\tau_{B}=4 \pi \eta R_{h}^{3} /\left(k_{B} T\right)$. At $T=100 \mathrm{~K}, \nu_{B}$ is expected to be decreased by a factor of 3 or even more if the viscosity $\eta$ increases with decreasing temperature. Similarly, the phase of the neutron scattering response was found to be shifted with respect to the inducing field from $2^{\circ}$ at $300 \mathrm{~K}$ to $15^{\circ}$ at $150 \mathrm{~K}$ and $20^{\circ}$ at $100 \mathrm{~K}$ [Fig. 4(d)], which indicates a continuous increase of the characteristic time $\tau_{B}$ of the Brownian rotation of particles as expected from Eq. (1).

This interpretation is in line with the ac susceptibility spectrum of this sample measured at room temperature at low perturbing ac fields $(1 \mathrm{mT})$ in a frequency range of $0.1 \mathrm{~Hz}-100 \mathrm{kHz}$, which demonstrates that the system responds very slowly to ac magnetic fields [see Fig. 9(a)]. The low-frequency limit, which should correspond to the equilibrium susceptibility $\chi^{\prime}=\chi_{0}=\mu \mu_{0} M_{S} /\left(k_{B} T\right)$, is not yet reached even at $0.1 \mathrm{~Hz}$, i.e., there is a considerable amount of moments, which at $100 \mathrm{~Hz}$ cannot keep up with the oscillating field. This is different compared to the spectrum of the $\mathrm{Fe}_{3} \mathrm{O}_{4}$-FF [Fig. 9(b)] where $10 \mathrm{~Hz}$ is already nearly in the low-frequency limit. An important difference that has to be mentioned, however, is that those susceptibility measurements were made in the low-field limit.

Superposition of a static field of $B_{\mathrm{st}}=20 \mathrm{mT}$ to an oscillating field of the same amplitude shows again the expected oscillation of the response with the same period as that of $B(t)$ [Fig. 7(d)]

\section{Field cooled in static magnetic field of $B_{\mathrm{st}}=20 \mathrm{mT}$}

Cooling down the Co-FF sample in a static field of $20 \mathrm{mT}$ to $100 \mathrm{~K}$ gives rise to an anisotropic scattering pattern resulting from the alignment of the field-induced structures formed above $T_{f}$. Once an alternating field is applied at 

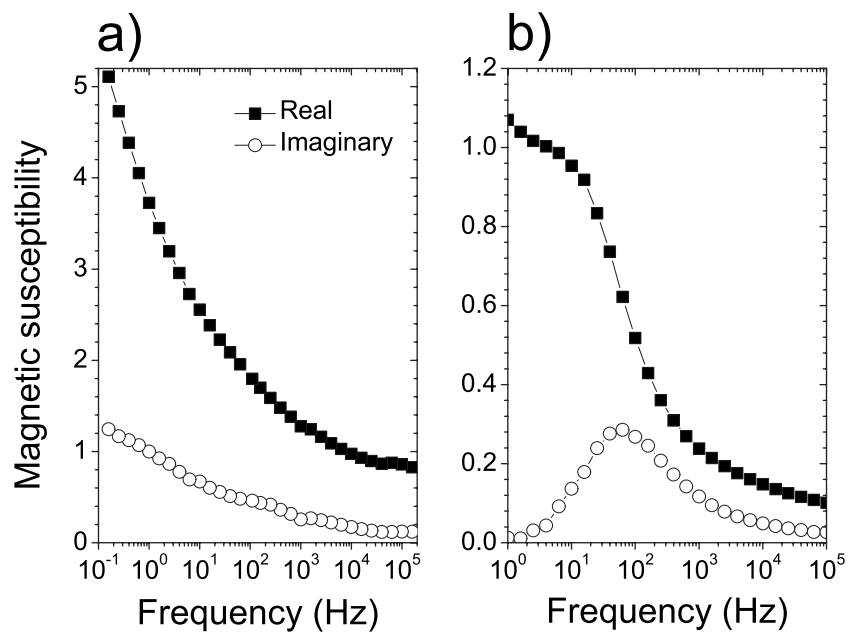

FIG. 9. Complex magnetic susceptibility for (a) Co-FF and (b) $\mathrm{Fe}_{3} \mathrm{O}_{4}$-FF.

$100 \mathrm{~K}$, some oscillations occur (Fig. 10). Basically, the dynamics is not different from that after zero-field cooling. The amount of freely rotating particles in FC is slightly lower than for ZFC and correspondingly the static contribution $\left(1-f_{e}\right) U(\alpha, \nu)$ is higher, in particular, for $\alpha=90^{\circ}$, which means that the immobilized particles are preferentially aligned along $B$. The field-induced structure factor $S(Q, \alpha$ $=0)$ is always lower than in ZFC.

\section{Co precipitates in solid $\mathrm{CuCo}$ alloy}

The SANS response of the CuCo solid alloy in an oscillating magnetic field is shown in Fig. 11 for three different frequencies. All curves could be perfectly fitted according to Eq. (6) by using the parameters of Table I and just by taking into account the damping resulting from the wavelength spread. No field-dependent interparticle structure factors had

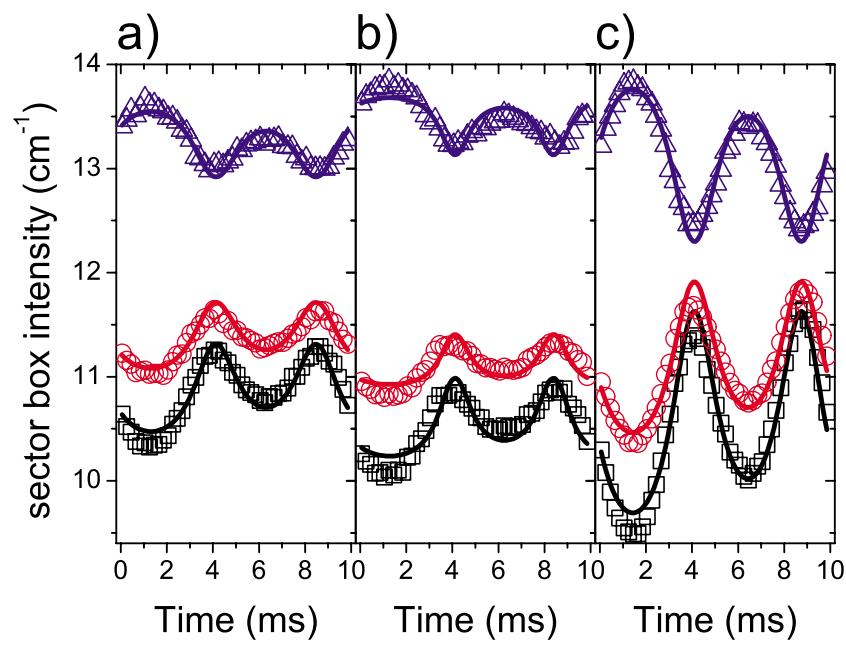

FIG. 10. (Color online) Oscillating behavior of Co-FF at $100 \mathrm{~Hz}$ and $B_{0}=20 \mathrm{mT}$ after cooling the sample in a static field of $20 \mathrm{mT}$ at (a) 100, (b) 150, and (c) $300 \mathrm{~K}$. For the sake of clarity, only one out of two measured points is shown, and static values are not shown but were included in the fit.

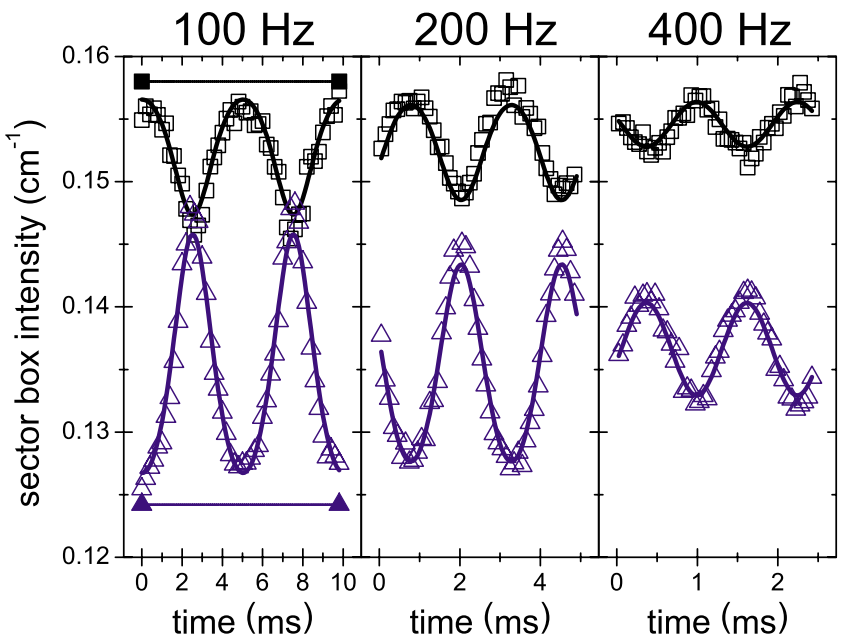

FIG. 11. (Color online) Room temperature SANS response of $\mathrm{CuCo}$ alloy at parallel (squares) and perpendicular (triangles) to the external field direction in an oscillating magnetic field of $B_{0}$ $=20 \mathrm{mT}$ at different frequencies (for the sake of clarity, only one out of two measured points is shown) and in a static field $B_{\text {st }}$ $=20 \mathrm{mT}$ (horizontal lines).

to be considered due to the very low concentration of Co precipitates. It turned out that for all frequencies up to $600 \mathrm{~Hz}$ all the magnetic particle moment follow the oscillating field, i.e., $f_{e}=1$ in Eq. (6) and no contribution of arrested moments occurs. Since particle rotation is excluded in the solid matrix, relaxation can only take place by the Néel mechanism of moment reorientation against the anisotropy energy $K_{a} V_{c}$.

Cooling down the sample to $120 \mathrm{~K}$ increased the argument $x$ in the Langevin function but no change in the volume fraction was observed. The same holds for a superposition of a dc field where the oscillating intensities have been explained by the same model, and no shift of the phase could be detected. This indicates that the characteristic time for Néel relaxation [Eq. (2)], i.e., for the moment rotation inside the particle, must be much shorter than $1 \mathrm{~ms}$ (the observable limit in this technique at $600 \mathrm{~Hz}$ ). Moreover, since even at $120 \mathrm{~K}$ there was no indication of moment blocking, the anisotropy $K_{a}$ cannot exceed $0.5 \times 10^{5} \mathrm{~J} \mathrm{~m}^{-3}$, which is by a factor of 5 lower than the bulk value of fcc-co reported in the literature. This could be due to the perturbations of the crystallographic structure at the surface of the nanoscaled particles.

The solid lines correspond to a model fit [Eq. (6)] using the parameters of Table I, which result a volume fraction of $100 \%$ for freely oscillating particle moment.

\section{DISCUSSION}

For both studied ferrofluid systems, the magnetic field oscillations induce scattering-intensity fluctuations. Once the oscillating field is switched on, larger structures are grown that become (partially) aligned when the field reaches its maximum valuegrow. Subsequently, the structures either completely disintegrate when the field direction changes ori- 
entation, or - if the frequency is high enough - the magnetic moments will change orientation and the particle positions will remain practically unchanged. The formation of these locally ordered domains in both dispersions is mainly governed by magnetic interactions between (single) dipolar chains, while the disintegration of these structures upon reducing the field is predominantly determined by diffusion. For instance, this diffusion-dominated disintegration of dipolar structures is the main reason for hysteresis effects in magnetization curves.

The maximum scattering intensity in the dynamic experiments does not reach the intensity obtained in static fields. This implies that on the time scale of the oscillations in the dynamic experiment $(<10 \mathrm{~ms})$ not all magnetic units become aligned. As is known from dynamic magnetic susceptibility experiments, the relaxation time strongly depends on the average cluster size. Therefore, it is mainly the presence of aggregates that prevents the scattering intensity from reaching full saturation in the oscillating field. The presented dynamic and static data can be quantitatively described when an effective dynamical structure factor that depends on the applied magnetic field by scaling with the square of the Langevin factor is incorporated. This is unambiguously shown in the $\mathrm{Fe}_{3} \mathrm{O}_{4}$-FF where above the melting point of the ferrofluid, the sector intensities in the direction parallel to the magnetic field oscillate in phase with the sector intensities perpendicular to the field. If the structure factor would have no effect on the applied field, then the scattering intensity in the $0^{\circ}$ direction would always be maximum in zero field.

Cooling the ferrofluid below the melting point of the carrier liquid results in an almost arrested dynamics. In $\mathrm{Fe}_{3} \mathrm{O}_{4}$-FF, Brownian rotation of the individual particles or structures is prevented and relaxation of the magnetic moments according to the Néel mechanism inside the particle cannot take place due to the anisotropy energy $K_{a} V_{c} \gg k_{B} T$. The effect of the temperature on the dynamical structure factor can be described quantitatively using the presented model. At temperatures far below the melting point of the solvent, the structure factor is close to unity, manifesting only relatively small structures if cooling is performed in zero field. Upon increasing the temperature, the dynamical structure factor increases and reaches a maximum just above the melting point in the direction perpendicular to the field. Subsequently, when the temperature, is increased to room temperature, the structure factor decreases again as a result of growing thermal fluctuations. The temperature dependency of the structure factor indicates that the formation of dipolar structures is mainly determined by the effective dipole-dipole interaction, which is enhanced by the (partial) alignment of the dipoles when an external magnetic field is applied. In the case of $\mathrm{Fe}_{3} \mathrm{O}_{4}$-FF, the dominating structure factor is $S\left(Q, \alpha=90^{\circ}\right)$, which reflects the formation of hexagons while for Co-FF at the same strength of magnetic field $S\left(Q, \alpha=0^{\circ}\right)$ is dominating, which reflects the presence of chainlike correlations. Furthermore, the number of particles that can follow the oscillations drops significantly with decreasing temperature due to the formation of larger structures with longer relaxation times and it tends to zero below $T_{f}$.

Clearly, "field-induced ordered domains" in ferrofluids cannot be considered as static units. Due to the fast rotation of individual particle moments and slow relaxation of larger domains, the system would end up fully arrested after a large number of oscillations. Our measurements showed instead that the process above $T_{f}$ is fully reversible and that there is no difference between the corresponding intensities measured at the beginning and at the end of the experiment. Instead, the domains have to be considered as "living objects": particles can move away from one domain by translational diffusion in the liquid and will be for some time free where in an ultimate oscillation cycle they will be stuck to a different domain. At a given temperature, the local ordering is characterized by the dynamical structure factors and the size of domains represents a time-averaged value corresponding to dynamical equilibrium. Below the freezing temperatures, the dynamics is almost fully arrested.

\section{CONCLUSION}

The results illustrate that the stroboscopic SANS technique allows the dynamics of structural environments of the magnetic nanoparticle dipoles in colloidal systems to be studied on a time scale well above the limitations of Neutron spin-echo techniques ${ }^{22}$ or Mössbauer spectroscopy ${ }^{23}$ $\left(10^{-6}-10^{-12} \mathrm{~s}\right)$ and complementary to $\mathrm{x}$-ray photon correlation spectroscopy ${ }^{24}$ and forced Rayleigh scattering. ${ }^{25}$ The effective dynamical structure factor depends on the magnetic field by scaling with the square of the Langevin factor. This shows that the dipolar structures are mainly determined by the effective dipole-dipole interaction, which is enhanced by the (partial) alignment of the dipoles in an external magnetic field. The dynamics of the field-induced ordering in the ferrofluid systems is governed by the fast Brownian rotation of individual nanoparticles and small aggregates while the magnetic relaxation of longer dipolar chains and local hexagonal domains is much slower. The stroboscopic SANS technique allowed elucidation of the dynamical nature of the locally ordered domains in both ferrofluids as living objects getting arrested below the freezing of the solvent. In contrast, no field-induced interparticle correlations occur in the diluted solid $\mathrm{CuCo}$ alloy where the moment relaxation is purely of fast Néel type, which-due to a low anisotropy constantfollows the oscillating field at all temperatures.

\section{ACKNOWLEDGMENTS}

This research has been supported by the European Commission under the sixth Framework through the Key Action: Strengthening the European Research Infrastructures, Contract No. RII-CT-2003-505925 and by the German Research Foundation DFG Project No. Wi-1151/3. 
*wiedenmann@ill.eu

${ }^{1}$ P. G. de Gennes and P. A. Pincus, Phys. Kondens. Mater. 11, 189 (1970).

${ }^{2}$ K. Butter, P. H. H. Bomans, P. M. Frederik, G. J. Vroege, and A. P. Philipse, Nat. Mater. 2, 88 (2003).

${ }^{3}$ M. Klokkenburg, B. H. Erné, J. D. Meeldijk, A. Wiedenmann, A. V. Petukhov, R. P. A. Dullens, and A. P. Philipse, Phys. Rev. Lett. 97, 185702 (2006).

${ }^{4}$ B. Groh and S. Dietrich, Phys. Rev. E 63, 021203 (2001).

${ }^{5} \mathrm{~S}$. Hess, in Physics of Complex and Supramolecular Fluids, edited by S. A. Safran and N. A. Clark (Wiley, New York, 1987), pp. 631-642.

${ }^{6}$ P. Ilg, (DOI: 10.1140/epje/i2007-10248-6) (to be published).

${ }^{7}$ A. Wiedenmann, A. Hoell, M. Kammel, and P. Boesecke, Phys. Rev. E 68, 031203 (2003).

${ }^{8}$ A. Wiedenmann and A. Heinemann, J. Magn. Magn. Mater. 289, 58 (2005).

${ }^{9}$ A. Wiedenmann, M. Kammel, A. Heinemann, and U. Keiderling, J. Phys.: Condens. Matter 18, S2713 (2006).

${ }^{10}$ M. Klokkenburg, B. H. Erné, A. Wiedenmann, A. V. Petukhov, and A. P. Philipse, Phys. Rev. E 75, 051408 (2007).

${ }^{11}$ A. Wiedenmann, U. Keiderling, R. May, and C. Dewhurst, Physica B 385, 453 (2006).

${ }^{12}$ A. Wiedenmann, U. Keiderling, K. Habicht, M. Russina, and R. Gähler, Phys. Rev. Lett. 97, 057202 (2006).
${ }^{13}$ R. Gähler and R. Golub, Z. Phys. B: Condens. Matter 56, 5 (1984).

${ }^{14}$ F. Luis, F. Petroff, J. M. Torres, L. M. Garcia, J. Bartolomé, J. Carrey, and A. Vaurès, Phys. Rev. Lett. 88, 217205 (2002).

${ }^{15}$ S. Morup and E. Tronc, Phys. Rev. Lett. 72, 3278 (1994).

${ }^{16}$ C. Djurberg, P. Svedlindh, P. Nordblad, M. F. Hansen, F. Bodker, and S. Morup, Phys. Rev. Lett. 79, 5154 (1997).

${ }^{17}$ W. Wagner, Z. Metallkd. 80, 873 (1989).

${ }^{18}$ B. W. M. Kuipers, I. A. Bakelaar, M. Klokkenburg, and B. H. Erné, Rev. Sci. Instrum. 79, 013901 (2008).

${ }^{19}$ J. Kohlbrecher, A. Wiedenmann, and H. Wollenberger, Z. Phys. B: Condens. Matter 104, 1 (1997).

${ }^{20}$ R. Pynn, J. B. Hayter, and S. W. Charles, Phys. Rev. Lett. 51, 710 (1983)

${ }^{21}$ S. Prévost, A. Wiedenmann, U. Keiderking, M. Klokkenburg, and B. Erné (unpublished).

${ }^{22}$ H. Casalta, P. Schleger, C. Bellouard, M. Hennion, I. Mirebeau, G. Ehlers, B. Farago, J. L. Dormann, M. Kelsch, M. Linde, and F. Phillipp, Phys. Rev. Lett. 82, 1301 (1999).

${ }^{23}$ M. F. Hansen, F. Bodker, S. Morup, K. Lefmann, K. N. Clausen, and P. A. Lindgard, Phys. Rev. Lett. 79, 4910 (1997).

${ }^{24}$ J. Lal, D. Abernathy, L. Auvray, O. Diat, and G. Grübel, J. Hydraul. Eng. 4, 263 (2001).

${ }^{25}$ J. C. Bacri, A. Cebers, A. Bourdon, G. Demouchy, B. M. Heegaard, and R. Perzynski, Phys. Rev. Lett. 74, 5032 (1995). 